\title{
Starch Sandstones in Building Bio-materials
}

\author{
Gabriel Mansour ${ }^{1}$, Maria Zoumaki ${ }^{1, *}$, and Dimitrios Tzetzis ${ }^{2}$ \\ ${ }^{1}$ Department of Mechanical Engineering, Laboratory of Machine Tools and Manufacturing \\ Engineering, Aristotle University of Thessaloniki, Greece \\ ${ }^{2}$ International Hellenic University, School of Science and Technology, Greece*
}

\begin{abstract}
A review of the recent literature shows that the use of more sustainable, eco-friendly recycled waste materials made from natural biopolymers is an important step of the planning process to reduce the environmental impacts of traditional building materials such as cement and concrete products. This study introduces the application of maize starch in the production of a novel biodegradable construction material. The samples prepared in this investigation were formed by heating a mixture of different proportions of starch, water and sand. The structural properties, morphology and chemical composition of materials were investigated by scanning electron microscopy (SEM) coupled with thermal gravimetric analysis (TGA). The structural characteristics and morphology of the study material to a certain extent resemble natural sandstones, the most common type of sedimentary rocks. Based on the uniaxial compressive strength classification schemes, comparing with the brittle deformation behavior of natural rocks, it can be considered that this material behaves as a polymer matrix composite with a ductile - thermoplastic mechanical behavior.
\end{abstract}

\section{Introduction}

Global warming has become an almost inevitable threat to human life and requires climate change adaptation and mitigation strategies with rapid reduction in global greenhouse gas emissions [1]. The construction sector produces a considerable amount of waste and greenhouse gas emissions that drive global warming. The recent literature on construction research indicates that biodegradable materials made from renewable sources are sustainable concrete alternatives that use natural polymers (such as starch and cellulose) instead of traditional cement [2-5].

The product developed by this project was obtained by heating a mixture of starch, water and sand. The strength and durability performances of such artificial starch sandstone were also investigated as a function of the composition materials. Based on the uniaxial compressive strength classification schemes, the mechanical behavior of the product can be characterized as a polymer - matrix composite with a more ductile fracture response compared to the brittle mechanical behavior of natural rock materials. In applied soil and rock mechanics, materials can be divided into natural building materials like sand, clay and natural rocks, and the artificial construction materials like cement, concrete and

${ }^{*}$ Corresponding author: mariazita687@gmail.com 
bricks. The materials can be also divided into two classes based on their relative behavior under stress conditions (when they fracture): The brittle solid materials under external loading exhibit little elastic deformation and negligible plastic deformation, while ductile materials under external loading exhibit negligible elastic deformation and substantial plastic deformation. Most building materials and natural rocks under stress (at laboratory temperature) behave as brittle materials with a tendency to break into smaller pieces [6]. The classification schemes of materials [7, 8] are generally based on three important engineering properties of rocks (uniaxial compressive strength, Young's modulus of elasticity and tensile strength) involving different types of compressive stress-strain curves, such as Type I: Elastic (e.g., basalts, quartzite, diabase, dolomites and strong limestones), Type II: Elastic - plastic (e.g., softer limestones, siltstone and tuff; type I or type II are usually typical of most traditional building materials, such as cement mortars, concrete and cement bricks), Type III: Plastic-elastic (e.g., sandstone, granites and schist cored parallel to the foliation), Type IV: Plastic-elastic-plastic (e.g., marbles and gneiss ), Type V: Plastic-elastic-sigmoid plastic (e.g., schist cored perpendicular to the foliation), Type VI: Elastic - plastic - creep (e.g., rock salts and other evaporite rocks) and Type VII: Ductile thermoplastic mechanical behavior (e.g., polymeric materials). Natural rocks based on their geological and engineering properties may be also classified into strong, moderate strong, medium and weak materials bounded by the uniaxial compressive strengths of $100,50,25$ and $15 \mathrm{MPa}$, respectively, with a ratio $(\mathrm{R})$ of tensile strength to compressive strength of approximately $0.23-0.41$ and a compressive modulus of elasticity of about $5-100 \mathrm{GPa}$ $[9,10]$. For example, moderate strong mudstone, moderate strong limestone, medium strong sandstone, strong limestone, strong sandstone and strong ironstone were found to have a compressive strength of about 50, 42, 85, 143, 168 and $190 \mathrm{MPa}$, respectively, with a ratio $\mathrm{R}$ of about $0.70,0.43,0.29,0.26,0.39$ and 0.23 , respectively, with typical values of uniaxial compressive modulus of elasticity for natural rocks (such as basalt, marble, granite, dolomite, limestone, sandstone and evaporite rocks) ranging from $5-100 \mathrm{GPa}$ [8- 11].

This study introduces the application of maize starch in the production of a novel biodegradable construction material formed by heating a mixture of starch with sand and water with good cost-effective and environmentally friendly properties and satisfactory compressive strength comparable to that of common red clay bricks. The strength and durability performances of the study material were also investigated as a function of the water to starch ratio. For each starch to sand ratio the maximum compressive strength corresponds to specific water content. Based on modulus of elasticity classification schemes for natural rocks, comparing with the brittle deformation behavior of natural rocks, it can be considered that this starch-sand composite behaves as a polymer - matrix composite with a ductile - thermoplastic mechanical behavior. One of the main objectives of this work is to validate the feasibility and accuracy of a novel preparation technique of this starch - sand material so that it can be easily transferred from the laboratory to an industrial scale.

\section{Materials and Methods}

In this study the starch - sandstone artificial material was prepared by heating a mixture of sand with corn starch and water (with constant sand to starch ratio of 5:1) according to two different heating techniques, using a microwave oven and a convection oven [5]. The cubic and cylindrical specimens of the starch-based material and natural rocks were illustrated in Figures 1a and 1b, respectively. The bio-composites based on thermoplastic starch and quartz sand were obtained by casting the above mixture in silicone rubber moulds (Figure 1c) and heated in a microwave oven (Daewoo, output power $800 \mathrm{~W}$, model KOR-6C17) for $5 \mathrm{~min}$, first at thaw mode for $1.5 \mathrm{~min}$ and then at full power mode for $3.5 \mathrm{~min}$ or heated at a 
temperature of $105{ }^{\circ} \mathrm{C}$ for $24 \mathrm{~h}$ in an industrial in an industrial convection oven. Uniaxial compressive strength test (Figure 1d) and Brazilian test (Figure 1e) were performed on cubic and cylindrical samples (at room temperature of about $20^{\circ} \mathrm{C}$ ) in a servo-controlled (M500-50AT Testometric tensile test machine), according to ISRM [12] suggested methods. The compressive Young's modulus of elasticity can be further determined as the slope of the tangent to the uniaxial compression stress-strain curve. It is generally taken at a stress level equal to $50 \%$ of the compressive strength. Tensile strength was further determined by an indirect test method, the Brazilian test, in which a diametrical line load is applied over the curved surface area of a cylindrical specimen having a length (L) - to diameter $(\mathrm{D})$ ratio $=1 / 2[6]$.

A disadvantage of the microwave heating procedure is the requirement to place a surcharge load (weight) above the samples in order to prevent deformation and the subsequent cracking and pop up of specimens while heating (Figure 1f). However, it was not considered necessary to place additional weight on the samples by the oven heating method [5]. This study introduces a novel preparation technique that will mitigate some of the problems above. The composites were placed in the oven by compressing the moulds between two flat Teflon plates, securely fastened together with Teflon screws (Figures $1 \mathrm{~g}, 1 \mathrm{~h}$ and 1i) in order to prevent the cracks and pop up of specimens while heating by the microwave heating method (Figure $1 \mathrm{j}$ ). The above method is suggested as an alternative technique designed to enable with insignificant cost the transition from the laboratory (Figure 1f) to production on an industrial scale (Figure 1j).

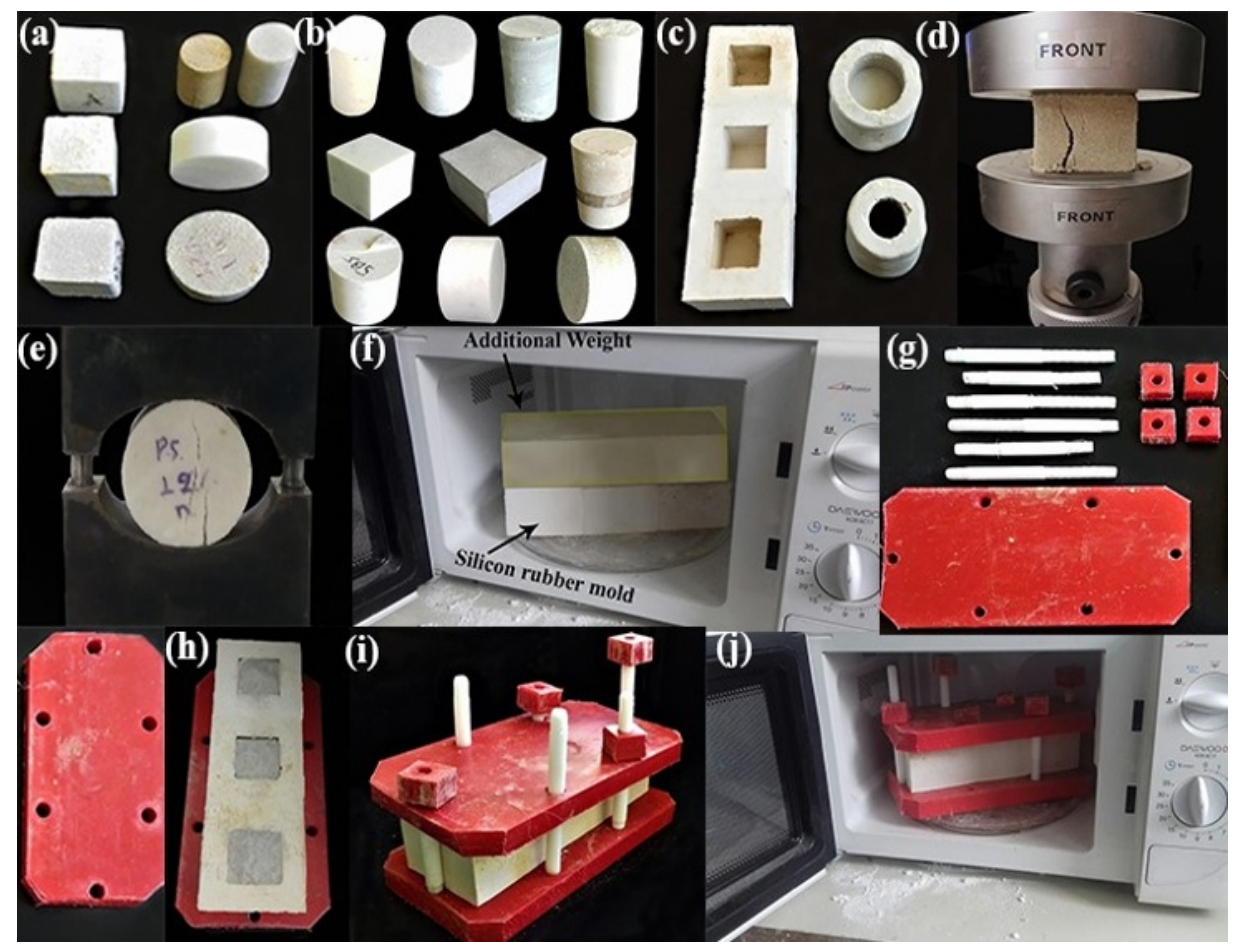

Fig. 1. (a),(b) Cubic and cylindrical specimens of the study material and natural rocks, (c) silicone rubber moulds, (d) uniaxial compressive strength test, (e) Brazilian test, (f) an additional weight over the samples while heating in a microwave and $(\mathrm{g})$ - (j) compressing the moulds between two flat Teflon plates (securely fastened together with Teflon screws) in order to prevent cracks and pop up of specimens while heating in a microwave oven. 


\section{Results and discussion}

This study deals with the use of maize starch as a bio-based construction material. The artificial sandstones prepared in this investigation were formed by heating a mixture of starch, water and sand in the microwave oven (Figure 1j). The uniaxial compressive strength of the prepared artificial solid material as a function of the composition of the mixture (with different proportions of water content and constant sand to starch ratio of 5:1) is presented in Figure 2. As shown in Figure 2, with an increase in water content, the compressive strength increases. At optimum water content of $12 \%$, the maximum compressive strength of $30 \mathrm{MPa}$ was achieved. However, a decrease in the compressive strength was observed above the optimum water content. A similar behaviour was observed for the stiffness of the testing specimens. The structural characteristics and chemical composition of materials were investigated by the scanning electron microscopy (SEM type JEOL JSM-840A, Japan). The mechanical properties and durability of the obtained solid starch-based materials were negatively affected by the highly porous structure morphology, as also confirmed by the SEM image (illustrated in Figure 3).

Thermogravimetric analysis (TGA) is a technique used for characterizing the thermal stability of a material [3]. The TGA curve in Figure 4 shows the mass loss percentage of a sample of this (starch - based) material as a function of increasing temperature (with constant heating rate of $5{ }^{\circ} \mathrm{C} / \mathrm{min}$, using a TGA analyzer TAQ50, TA Instruments, USA). The first mass change appears at around $100{ }^{\circ} \mathrm{C}$, which is associated with the loss of the absorbed water (in the structure of the investigated material) at low temperatures in the range of $90-150{ }^{\circ} \mathrm{C}$. With further heating, the removal of moisture becomes difficult because water molecules are trapped within the gelatinized starch matrix. The result clearly indicates that the greater mass change is associated with the loss of starch content. This mass loss coincides with the thermal degradation of starch matrix at temperatures above $200{ }^{\circ} \mathrm{C}$ with a maximum mass loss rate at around $290{ }^{\circ} \mathrm{C}$ (the burning temperature of native corn starch) and the degradation ended at a temperature of $450{ }^{\circ} \mathrm{C}$. The technique of preparing this type of solid polymeric material uses gelatinized corn starch as a binder and sand as filler, and therefore it morphologically resembles an artificial sandstone material. Most building materials and natural rocks after compression tests tend to break into smaller pieces and therefore are classified as brittle materials (types I - V) in Figure 5a. However, as evident from Figure $5 \mathrm{~b}$, the evaporitic rock salt revealed the characteristic plastic creeping deformation before failure (type VI) and the artificial starch - sandstone material exhibited the typical thermoplastic behaviour of polymeric materials (type VII).

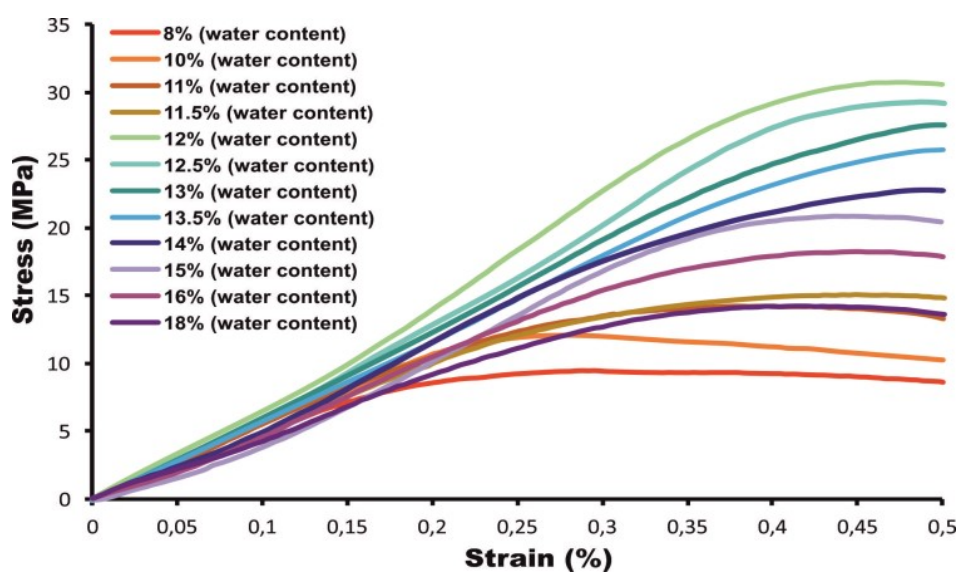

Fig. 2. The uniaxial compressive strength of the study material as a function of water content (with constant sand to starch ratio 5:1) 


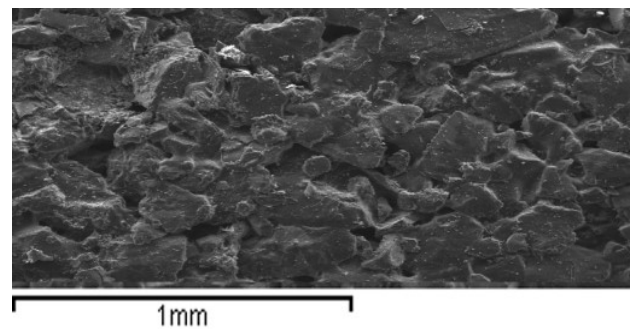

Fig. 3. SEM image of the structural characteristics of the obtained starch-based solid material

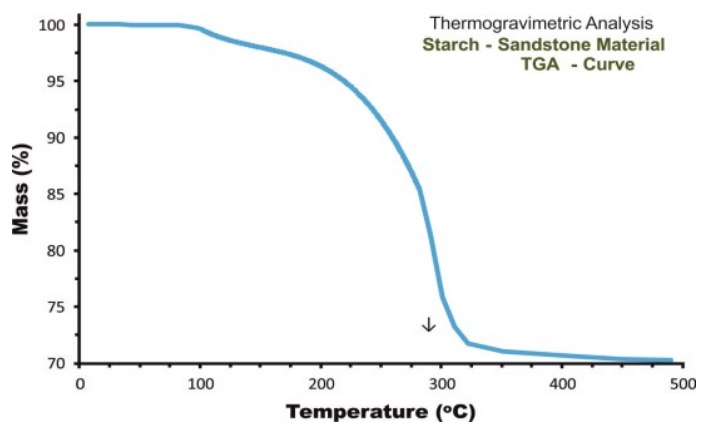

Fig. 4. The TGA curves of the study starch - sandstone material
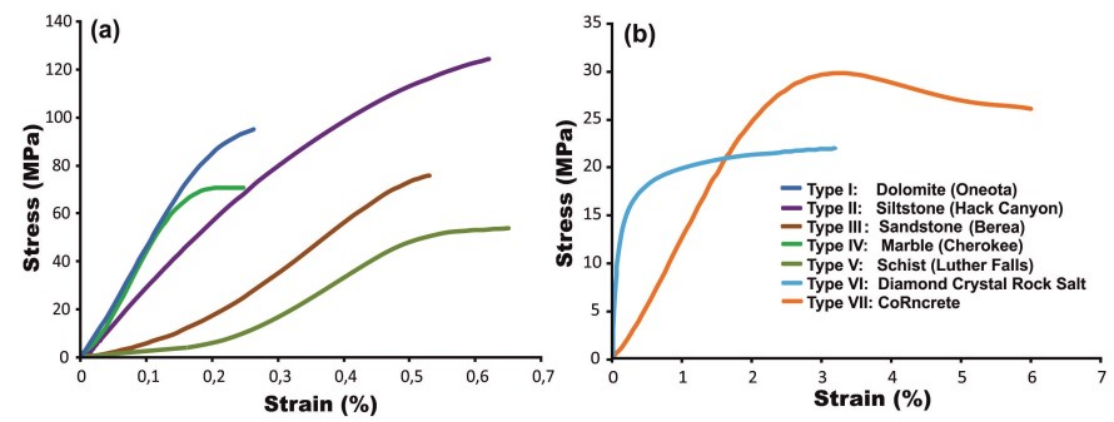

Fig. 5. Stress-strain curves for different types of materials in uniaxial compression strength test to failure.

Samples of the study material were tested and found to have a compressive strength of about $30 \mathrm{MPa}$ with significantly low values of compressive modulus of elasticity (less than $2 \mathrm{GPa}$ ) and a ratio $(\mathrm{R})$ of tensile strength to compressive strength of approximately 0.20 . On the other hand, the most common types of building materials (such as concrete and cement mortars) and natural rocks, in general, tend to have a tensile strength of $1 / 10$ of their compressive strengths $[13,14]$. As can be seen in the representative examples illustrated in Figure 5, the uniaxial compressive strength ranges from about 20 to $130 \mathrm{MPa}$, and the compressive modulus of elasticity ranges from about $13 \mathrm{GPa}$ to about $50 \mathrm{GPa}$, while the starch - sandstone is characterized by a very low value of elastic modulus of about 1.5 GPa.

\section{Conclusions}

The technique of preparing this type of solid polymeric material is based on the physical modification of starch and the formation of a gelatinized starch matrix that binds sand grains and forms a rapid hardening composite structure. The most important factors that 
were found to affect the physical and mechanical properties of this starch-based composition were the proportions of starch, water and sand, heating scheme, sand particle size distribution, maize starch gelatinization process and the bonding of starch matrix with sand grains. A maximum uniaxial compressive strength was obtained at the optimum water - starch ratio corresponding to the maximum dry density. Generally, this product exhibits similar physical properties with cement mortars as well as some common types of natural rocks and morphologically resembles an artificial sandstone material. The test results showed that this type of novel construction material exhibited the typical thermoplastic behaviour of polymer - matrix composite materials, however as the starch to sand ratio gets smaller, the plastic zone deformation reduces, and the failure becomes more brittle.

This research is co-financed by Greece and the European Union (European Social Fund- ESF) through the Operational Programme «Human Resources Development, Education and Lifelong Learning» in the context of the project "Strengthening Human Resources Research Potential via Doctorate Research" (MIS-5000432), implemented by the State Scholarships Foundation (IKY).

\section{References}

1. Daniel Oudin Åströml, D.O., Forsberg B., Ebi K.L., Rocklöv J., Nat. Clim. Change 3, 1050-1054 (2013).

2. Zoumaki, M., Tzetzis, D., Mansour, G., IOP CONF. SER. MATER. SCI. ENG 564, 012037 (2019).

3. Mansour, G., Zoumaki, M., Marinopoulou, A., Raphaelides, S., Tzetzisc, D., Zoumakis, N., Starke 72, 1900166 (2020).

4. Le, A.T., Gacoin, A., Li, A., Mai, T.H., Rebay, M., Delmas, Y, Constr Build Mater. 61, 106-113 (2014).

5. Kulshreshtha, Y., Schlangen, E., Jonkers, H.M., Vardon, P.J., LA Van Paassen, L.A., Constr Build Mater. 154, 411-423 (2017).

6. Johnson, R.B., Degraff, J.V. Principles of Engineering Geology (John Wiley \& Sons, New York 1988).

7. Miller, R.P., (Engineering classification and index properties for intact rock, Ph.D., Thesis, Univ. Illinois 1965).

8. Tatsuoka, F., Shibuya,S., Proceedings of the $9^{\text {th }}$ Asian Regional Conference on SMFE, Bangkok, 101-170 (1992).

9. Agustawijaya, D.S., Civ. Eng. Dimens. 9, 9-14 (2007).

10. Kanji, M.A., J. Rock Mech. Geotech. Eng. 6, 186-195 (2014).

11. Dobereiner, L., De Freitas, M.H., Geotechnique 36(1), 79-94 (1986).

12. ISRM Suggested Methods Rock characterization testing and monitoring (Editor: E.T. Brown, Published for the commission on testing methods, International Society for Rock Mechanics, Pergamon Press 1981).

13. Sari, M., Glob. j. Earth sci. eng. 5, 1-25 (2018).

14. Stagg, K.G., Zienkiewicz, O.C., Rock Mechanics in Engineering Practice (John Willey \& Sons, USA 1975) 